\title{
Valproic acid-induced histone acetylation suppresses CYP19 gene expression and inhibits the growth and survival of endometrial stromal cells
}

\author{
YU CHEN, SHENGYUN CAI, JINGWEN WANG and MINGJUAN XU \\ Department of Obstetrics and Gynaecology, Changhai Hospital, \\ The Second Military Medical University, Shanghai 200433, P.R. China
}

Received January 26, 2015; Accepted June 12, 2015

DOI: $10.3892 /$ ijmm.2015.2263

\begin{abstract}
Endometriosis is a common type of estrogen-dependent, gynecological and chronic inflammatory disease. Epigenetics refers to changes in gene expression that occur without altering the DNA sequence or DNA content. Histone modification dominates epigenetics, and histone acetylation is the most extensively studied type of histone modification. The CYP19 gene is the gene that encodes P450 aromatase, which regulates the synthesis of estrogen. Hence, we conducted this study to investigate whether histone acetylation has an effect on CYP19 expression and whether histone acetylation is related to endometrial stromal cells (ESCs). Reverse transcriptionquantitative polymerase chain reaction (RT-qPCR), western blot analysis and chromatin immunoprecipitation assays were performed. The results revealed that valproic acid (VPA) significantly promoted histone acetylation in the ESCs, which inhibited histone acetylation in the promoter region of the CYP19 gene, thus suppressing its expression. We also noted that VPA inhibited cell viability and proliferation, and induced the apoptosis, of ESCs. The findings of our study on histone acetylation, endometriosis and the $C Y P 19$ gene provide insight which may aid in the research of histone acetylation and suggest that the CYP19 gene may be a novel therapeutic target and method for the treatment of endometriosis.
\end{abstract}

Correspondence to: Dr Mingjuan Xu, Department of Obstetrics and Gynaecology, Changhai Hospital, The Second Military Medical University, 168 Changhai Road, Shanghai 200433, P.R. China

E-mail: mingjuanxush@163.com

Abbreviations: VPA, valproic acid; HATs, histone acetyltransferases; HDACs, histone deacetylases; HDACis, histone deacetylase inhibitors; ESCs, endometriotal stromal cells; NESCs, normal endometrial stromal cells

Key words: valproic acid, CYP19, endometriosis, endometrial stromal cells, histone acetylation

\section{Introduction}

Endometriosis, which is a benign and estrogen-dependent disease, is the most common chronic inflammatory gynecological disease in women of reproductive age, and it is mainly caused by the ectopic presence and growth of normal endometrial cells in the pelvic cavity instead of the uterine cavity $(1,2)$. The morbidity associated with endometriosis is approximately $10 \%$ in women of child-bearing age, and this increases to 20-30\% in women with subfertility and to $40-60 \%$ in women with dysmenorrhea (1-3). The most common symptoms are severe dysmenorrhea, dyspareunia, pelvic pain and infertility (4), which markedly reduce the quality of life of affected women.

The goal of current medical treatments is to inhibit the effects of estrogen on ectopic implants through the suppression of the production of ovarian estrogenthrough oral contraceptives, aromatase inhibitors, androgenic agents and gonadotropin-releasing hormone analogues $(1,2,5,6)$. Anti-estrogen hormonal therapies can be prescribed only for a short period of time (6-9 months) due to the undesirable side-effects, such as bone density loss, pseudomenopause, hot flushes, mood swings, an increased risk of uterine and ovarian cancers, and compromised pregnancy, which profoundly affect the quality of life and emotional and physical wellbeing of patients with endometriosis $(1,2,5,6)$. Surprisingly, the disease re-establishes at a rate of approximately 50-60\% within a year, after the cessation of anti-estrogen therapy $(5,6)$. Hence, the discovery of a non-estrogen or non-steroidal therapeutic target, such as histone modification, which controls the growth and survival of endometrial stromal cells (ESCs), is urgently required for the treatment of endometriosis.

Epigenetics, a relatively new field of study, focuses on investigating the stable inheritance of phenotypes of cells and organisms which occur without changes in the DNA sequence or DNA content (7). Epigenetic phenotypes can be conferred through nuclear processes, such as DNA methylation and chromatin modifications (e.g., acetylation, biotinylation, isomerization, methylation, phosphorylation, ribosylation, sumoylation and ubiquitination of histones), and they underlie the regulation of all genome functions, including gene expression, DNA replication and genome stability $(8,9)$. Accumulating evidence indicates that several epigenetic aberrations are involved in the pathogenesis of endometriosis (10-12). 
Histone modification is the main mechanism of epigenetics, and it serves to regulate gene expression following transcription without altering the sequence of the silenced genes. Modifications to histones at $\mathrm{N}$-terminal histone tails, which protrude from the nucleosomes, have been recognized as markers of genes undergoing epigenetic abnormality in diseases (13). At least 8 patterns of histone modification have been identified, and acetylation has been the most intensively studied (14). Histone acetylation is a reversible situation that can either disrupt chromosomal contacts or affect non-histone protein interactions with chromatin, thus altering chromatin structure and gene expression (15). Histone acetyltransferases (HATs) and histone deacetylases (HDACs) acetylate and deacetylate lysine residues on the N-terminal region of histone proteins, which regulate the access of transcriptional factors to DNA and consequently regulate gene expression. Histone deacetylases inhibitors (HDACis) appear to significantly promote histone acetylation (11). Several HDACis have been identified, such as trichostatin A (TSA), sodium butyrate $(\mathrm{NaB})$ and valproic acid (VPA) (16). However, the effects of histone acetylation on the CYP19 gene have not yet been fully investigated.

The CYP19 gene encodes P450 aromatase, and cyclooxegenase-2 (COX2) increases the expression of $\mathrm{P} 450$ aromatase (17-19). P450 aromatase catalyzes the final steps in the biosynthesis of estrogen from androgens in two ways: i) from androstenedione to estrone and ii) from testosterone to estradiol (20). Thus, the aromatase cytochrome P450 is involved in the final and rate-limiting step of estrogen synthesis and is associated with circulating estrogen levels (21). Studies have indicated that aromatase activity does not occur in endometrial tissue from women without uterine diseases. By contrast, endometrial lesions express aromatase, which is accompanied by increased mRNA levels, and aromatase enzyme activity is detectable in endometriosis $(18,22)$. The enzyme aromatase $\mathrm{P} 450$ is aberrantly expressed in patients with endometriosis, and this results in the production of estrogen in endometrial lesions. Furthermore, estrogen promotes the secretion of several inflammatory cytokines and growth factors, which contributes to the progression of endometriosis and stimulates estrogen production (23).

In the present study, we first identified normal endometrial stromal cells (NESCs) and ESCs. We then promoted histone acetylation in the ESCs through VPA. Subsequently, we measured cell viability, proliferation and apoptosis. Finally, ChIP assay, reverse transcription-quantitative polymerase chain reaction (RT-qPCR) and western blot analysis were performed to investigate the effects of histone acetylation on CYP19 expression. The results demonstrated that histone acetylation inhibited the viability and proliferation of the ESCs whilst promoting apoptosis, possibly by downregulating CYP19 expression. This discovery may prove to be of use in the targeted therapy of endometriosis.

\section{Materials and methods}

Cell culture and identification. Human NESCs and ESCs were kindly donated by the School of Medicine, Shanghai Jiao Tong University (Shanghai, China). The ESCs and NESCs were cultured in DMEM/F12 (HyClone, Logan, UT, USA) supplemented with $10 \%$ fetal bovine serum (FBS), $100 \mathrm{U} / \mathrm{ml}$ penicillin and $100 \mathrm{mg} / \mathrm{ml}$ streptomycin (Sigma-Aldrich, St. Louis, MO,
USA) in an atmosphere of $5 \% \mathrm{CO}_{2}$ at $37^{\circ} \mathrm{C}$. In the monolayer culture, and as previously described, the cells were identified by immunofluorescence staining using vimentin, cytokeratin (24) and prolactin (PRL) (25).

Immunofluorescence staining. After the third passage and when the ESCs reached approximately $80 \%$ confluence, they were fixed in $4 \%$ paraformaldehyde on ice. They were then washed 3 times with phosphate-buffered saline (PBS) and blocked with $10 \%$ horse serum. Subsequently, the samples were incubated overnight with primary antibodies (Santa Cruz Biotechnology, Inc., Santa Cruz, CA, USA), including mouse monoclonal vimentin (sc-6260), mouse monoclonal cytokeratin (sc-57004) and goat polyclonal PRL (sc-7805) at $4^{\circ} \mathrm{C}$. After the cells had been again washed 3 times, the cell slides were incubated at room temperature with the goat anti-mouse (A-11017) and rabbit anti-goat (A-11078) secondary antibodies (Invitrogen, Carlsbad, CA, USA) for $30 \mathrm{~min}$. Images were captured using a laser confocal microscope (FV1000; Olympus, Tokyo, Japan).

Treatment with VPA. To enhance the acetylation of histones, the ESCs were digested and plated in $10-\mathrm{cm}$ dishes (Corning, Inc., Tewksbury, MA, USA), followed by incubation for $24 \mathrm{~h}$ with VPA (8 mM; Sigma-Aldrich). Untreated cells served as controls.

Chromatin immunoprecipitation (ChIP) assay. ChIP assay was performed using a Pierce Agarose ChIP kit (Pierce Biotechnology, Rockford, IL, USA) according to the manufacturer's instructions. The immunoprecipitated DNA was subjected to PCR analysis of the promoter regions of the CYP19 gene. The sequences of the primers for the ChIP assay were as follows: $C Y P 19$ sense, 5'-AGT AA CACA GAA CAG TTG CA-3' and antisense, 5'-TCC AGA CTC GCA TGA ATT CTC CGT A-3', and the product was $188 \mathrm{bp}$. The PCR conditions were as follows: $94^{\circ} \mathrm{C}$ for $10 \mathrm{~min}$, followed by 35 cycles of $94^{\circ} \mathrm{C}$ for $30 \mathrm{sec}, 55^{\circ} \mathrm{C}$ for $30 \mathrm{sec}$, and $72^{\circ} \mathrm{C}$ for $30 \mathrm{sec}$, with a final extension at $72^{\circ} \mathrm{C}$ for $10 \mathrm{~min}$, using a PCR amplification kit (Takara Biotechnology, Dalian, China). The PCR products were analyzed by $2 \%$ agarose gel electrophoresis.

Transfection with small interfering RNA (siRNA). The siRNA was synthesized by GenaPharma Co. (Shanghai, China). Briefly, $5 \mu$ l of siRNA (CYP19 siRNA, sc-41498; Santa Cruz Biotechnology, Inc.), $10 \mu \mathrm{l}$ of Lipofectamine 2000 (Invitrogen) and $245 \mu \mathrm{l}$ of non-serum DMEM were mixed, followed by incubation for $30 \mathrm{~min}$ at room temperature. The mixtures were then equally distributed into the 6-well cultured cells followed by incubation at $37^{\circ} \mathrm{C}$ for transfection into the cells. Cells transfected with the control siRNA (sc-37007; Santa Cruz Biotechnology, Inc.) instead of the CYP19 siRNA were used as the negative control.

MTT assay. Cell viability was evaluated by MTT assay. Briefly, the cells were digested and re-seeded into evenly 96 -well plates. Subsequently, $20 \mu 1 \mathrm{MTT}$ solution $(5 \mathrm{mg} / \mathrm{ml})$ were added to the medium, and the cells were incubated for $4 \mathrm{~h}$ at $37^{\circ} \mathrm{C}$. The mixtures were then centrifuged at 8,000 rpm for $15 \mathrm{~min}$ and the supernatant was discarded. The formazan crystals were dissolved in dimethyl sulfoxide (DMSO; Sigma-Aldrich). The 
absorbance of the samples was measured at $490 \mathrm{~nm}$ using an EnVision ${ }^{\circledR}$ Multilabel Reader (PerkinElmer, Waltham, MA, USA).

5-Bromo-2-deoxyuridine (BrdU) assay. The proliferation of the ESCs was investigated by BrdU staining using BrdU enzyme-linked immunosorbent assay (ELISA) kit (colorimetric; Roche Diagnostics GmbH, Penzberg, Germany). Briefly, $5 \times 10^{3}$ cells/well were seeded into 96-well plates. When the cells grew to a confluence of $50 \%$, the medium was discarded and fresh BrdU-medium was added. Subsequently, the cells were incubated in $5 \% \mathrm{CO}_{2}$ at $37^{\circ} \mathrm{C}$ for $60 \mathrm{~min}$. After being washed with PBS and fixed with $70 \%$ ethanol, the cells were incubated with mouse monoclonal BrdU antibody (sc-32323 FITC; Santa Cruz Biotechnology, Inc.). The cells were observed and counted in randomly selected fields under a fluorescence microscope (Olympus). Data were presented as percentages of BrdU-positive cells vs. the total cell percentage.

Hoechst staining and terminal deoxynucleotidyl transferase-mediated dUTP nick-end labeling (TUNEL) assay. For Hoechst staining, $1 \mathrm{ml}$ of Hoechst 33258 staining solution $(10 \mu \mathrm{g} / \mathrm{ml}$; Beyotime, Nanjing, China) was added to the medium, and the cells were incubated at room temperature for 5 min. Subsequently, after the Hoechst medium was discarded and the cells were washed with PBS, fluorescence mounting liquid (Beyotime) was added. The apoptotic rate was calculated as the number of apoptotic cells/total number of cells $\mathrm{x} 100 \%$. For the TUNEL, the cells were fixed with $4 \%$ paraformaldehyde for $10 \mathrm{~min}$ at room temperature and permeabilized with ice-cold $0.1 \%$ Triton X-100 for $10 \mathrm{~min}$. Cell apoptosis was evaluated using a TUNEL Apoptosis Detection kit (Merck Millipore, Billerica, MA, USA) following the manufacturer's instructions. All cells were observed under a fluorescence microscope (Olympus). The positive cells were counted in randomly selected fields, and the number was averaged for further analysis.

$R T-q P C R$. Total RNA was isolated using TRIzol reagent (Invitrogen). Reverse transcription of the RNA (1 mg) into complementary DNA (cDNA) was conducted using the QuantiTect Reverse Transcription kit (Qiagen, Courtaboeuf, France). Subsequently, quantitative PCR (qPCR_ was performed using the QuantiTect SYBR-Green RT-PCR kit (Qiagen) with the StepOnePlus ${ }^{\mathrm{TM}}$ Real-Time PCR system (Life Technologies, Rockville, MD, USA). The CYP19 mRNA levels were measured by RT-qPCR. $\beta$-actin served as a reference gene. All samples were tested in triplicate. Data were analyzed using the comparative threshold cycle method $\left(2^{\Delta \Delta} \mathrm{Ct}\right)$.

Western blot analyisis. The cells were digested using $1 \%$ trypsin-ethylenediaminetetraacetic acid (EDTA) and harvested. Subsequently, the cell lysates were centrifuged, and then mixed with radioimmunoprecipitation assay (RIPA) buffer (Sigma-Aldrich). The mixture was occasionally shaken on ice to release the protein. The protein concentration was determined using Bio-Rad Protein Analysis kits (Bio-Rad, Hercules, CA, USA). Target proteins were isolated and transferred onto polyvinylidene difluoride (PVDF) membranes by sodium dodecyl sulfate-polyacrylamide gel electrophoresis (SDS-PAGE). The transferred proteins were incubated with primary antibodies (all from Santa Cruz Biotechnology, Inc.), including goat polyclonal acetylated histone H3 (Lys9, sc-8655; diluted at 1:1,000, v/v), rabbit polyclonal acetylated histone H4 (Lys12, sc-8661-R; 1:1,000), goat polyclonal CYP19 (sc-14245; 1:500) and rabbit polyclonal $\beta$-actin (sc-130656; 1:1,000) antibodies. The PVDF membranes were incubated with horseradish peroxidase (HRP)-conjugated secondary antibody (diluted at 1:5,000, v/v; Santa Cruz Biotechnology, Inc.), followed by treatment with chemiluminescence substrate (Pierce Biotechnology). The blots were observed and photographed using an ImageQuant LAS 4000 biomolecular imager (GE Healthcare, Piscataway, NJ, USA), and densitometric analysis was carried out using Quantity One software (Bio-Rad).

Statistical analysis. Data are presented as the means \pm SD of at least 3 independent experiments. The differences between 2 groups were examined using the Student's t-test, while the differences between 3 or more groups were compared by one-way analysis of variance (ANOVA). Values of $\mathrm{P}<0.05$ and $\mathrm{P}<0.01$ were considered to indicate statistically significant differences.

\section{Results}

ESCs are positive for vimentin, cytokeratin and PRL, whereas NESCs are not. As ESCs are characterized by the positive expression of vimentin, cytokeratin and PRL (26), both the ESCs and NESCs were subjected to immunofluorescence staining for the detection of the expression of these markers. As shown in Fig. 1, fluorescence staining for vimentin, cytokeratin and PRL was visible in the cytoplasm of the ESCs, but was absent in the NESCs (Fig. 1).

Histone acetylation is decreased in ESCs. The differences in the expression of acetylated histones between the ESCs and NESCs were further examined by western blot analysis. The results revealed that the ESCs had a significantly lower expression of acetylated histone $\mathrm{H} 3$ and $\mathrm{H} 4$ compared with the NESCs (Fig. 2A). The expression of acetylated histone $\mathrm{H} 3$ and $\mathrm{H} 4$ in the NESCs was 7.9-fold (Fig. 2B) and 18.2-fold (Fig. 2C) greater, respectively, compared with that in the ESCs. These results confirm the differences between ESCs and NESCs, and the following experiments were conducted using the ESC model.

VPA enhances the acetylation of histone $\mathrm{H3}$ and $\mathrm{H} 4$ in the ESCs. To investigate whether VPA alters the acetylation of histones in ESCs, we evaluated the global acetylation of histone $\mathrm{H} 3$ and $\mathrm{H} 4$ by western blot analysis. Low levels of acetylated histone $\mathrm{H} 3$ and $\mathrm{H} 4$ were detected in the control group (Fig. 3). By contrast, the levels of acetylated histone $\mathrm{H} 3$ and acetylated histone $\mathrm{H} 4$ in the ESCs were significantly increased by treatment with VPA. The levels of acetylated histone $\mathrm{H} 3$ and $\mathrm{H} 4$ in the VPA-treated ESCs were 8.7-fold (Fig. 3B) and 6.9-fold (Fig. 3C) greater, respectively, compared with those of the control group, thus demonstrating that VPA promotes histone acetylation.

Histone acetylation is reduced in the promoter region of the CYP19 gene by VPA. To examine the effects of VPA on the 

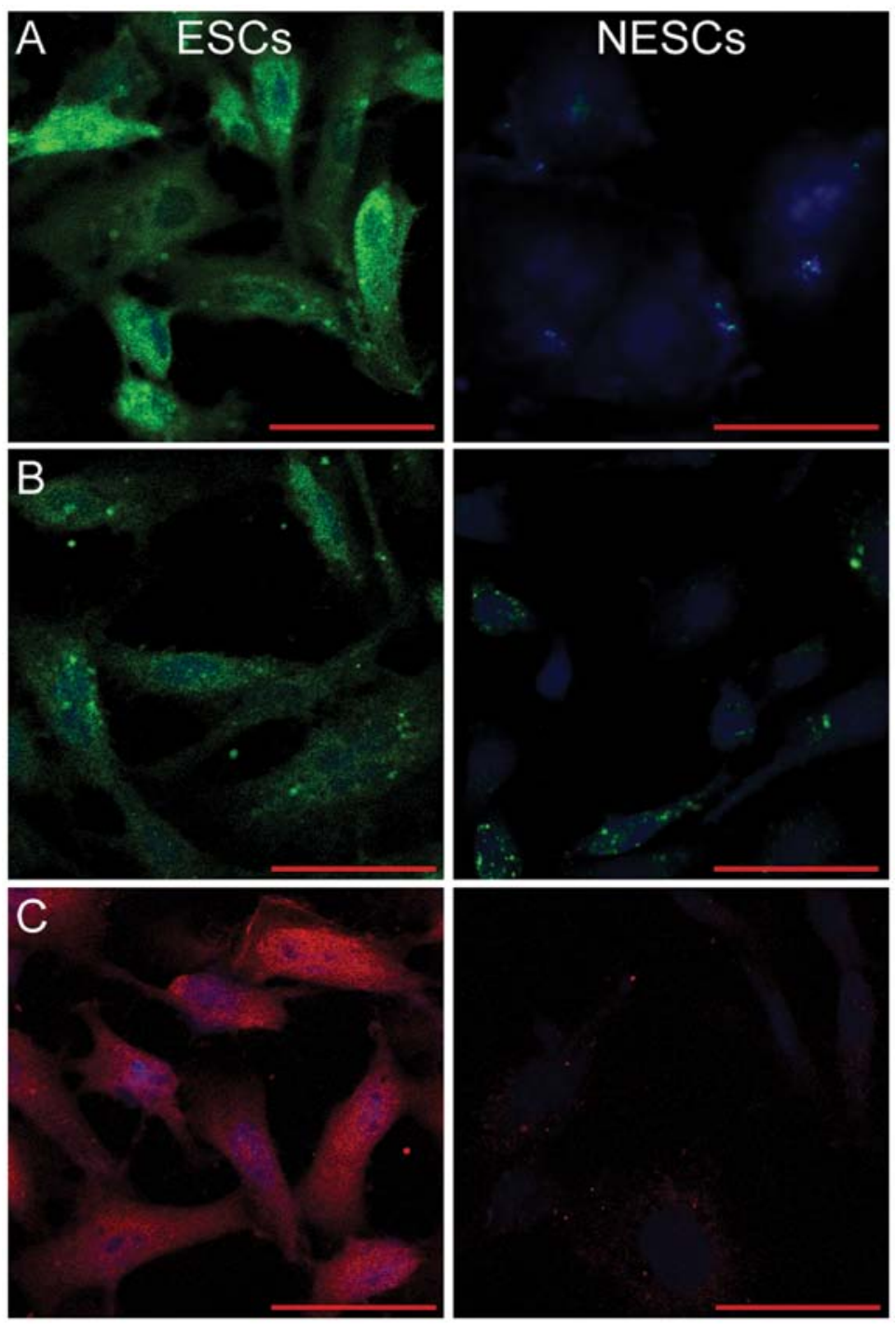

Figure 1. Immunofluorescence staining for vimentin, cytokeratin and prolactin (PRL) in endometrial stromal cells (ESCs) and normal endometrial stromal cells (NESCs). (A) Cytokeratin, (B) PRL, (C) vimentin. n=3; scale bars, $100 \mu \mathrm{m}$.

acetylation of the promoter region of the CYP19 gene in ESCs, ChIP assay with antibodies against acetylated histone H3 and acetylated histone $\mathrm{H} 4$ was performed. The immunoprecipitated DNA from the untreated and VPA-treated ESCs was isolated and subjected to PCR using primers for the promoter regions of the CYP19 gene, and a 188-bp fragment was amplified (Fig. 4). VPA significantly decreased the amounts of PCR product, suggesting that the acetylation of histone $\mathrm{H} 3$ and $\mathrm{H} 4$ was reduced in the promoter region of the CYP19 gene in the VPA-treated ESCs (Fig. 4).

CYP19 expression is suppressed by VPA. To investigate the expression of CYP19 at the mRNA and protein level following treatment with VPA, RT-qPCR and western blot analysis were performed. The relative mRNA expression of CYP19 in the VPA-treated ESCs $(1.42 \pm 0.38)$ was significantly decreased compared with that of the control group $(8.83 \pm 1.02$; Fig. 5A). Similarly, at the protein level, CYP19 expression was inhibited by VPA (Fig. 5B) and a 6.1-fold decrease was noted, compared with the control group (Fig. 5C). These results confirm that VPA inhibits CYP19 expression.
VPA and CYP19 knockout co-inhibit the viability and proliferation of ESCs. The effects of VPA and CYP19 on the viability and proliferation of the ESCs were investigated by MTT assay and BrdU assay, respectively. The viability of the VPA-treated ESCs was significantly decreased compared with that of the control group (Fig. 6A). VPA significantly inhibited BrdU incorporation into the ESCs. BrdU incorporation decreased to $20.7 \pm 2.52 \%$ relative to the control group following treatment with VPA at $8 \mathrm{mM}$ (Fig. 6B). Furthermore, we also noted that CYP19 siRNA inhibited the viability (Fig. 6A) and proliferation (Fig. 6B) of the ESCs compared with the control siRNA group and even enhanced the inhibitory effects of VPA.

VPA and CYP19 co-induce the apoptosis of ESCs. To investigate the effects of VPA and CYP19 siRNA on ESC apoptosis, Hoechst staining and TUNEL assay were performed. The percentage of apoptotic cells in the VPA-treated group increased to $34.4 \pm 2.89 \%$, and in the CYP19 siRNA group, it increased to $43.5 \pm 1.49 \%$, which was a significant increase compared with the control and control siRNA groups, respectively (Fig. 7A). Similarly, the results from TUNEL assay results also revealed a 
A

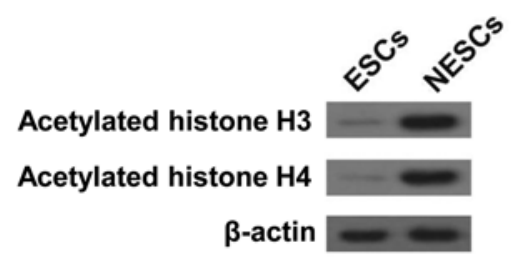

B Acetylated histone $\mathrm{H3}$

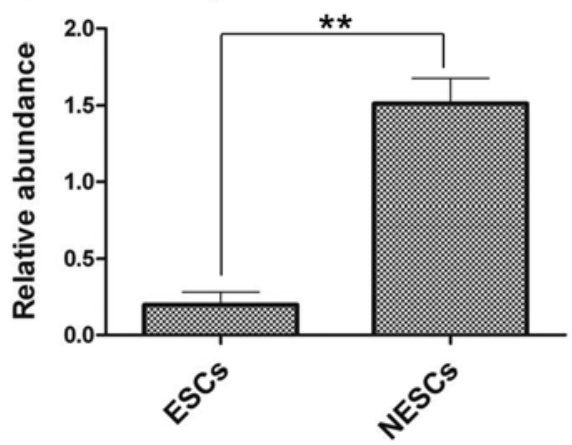

C Acetylated histone $\mathrm{H4}$

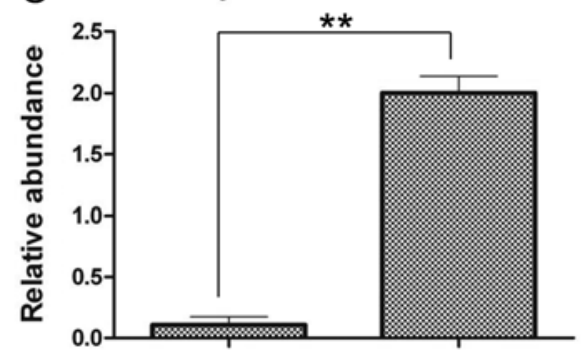

$\varepsilon^{c^{s}}$

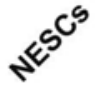

Figure 2. (A) Western blot analysis of acetylated histone $\mathrm{H} 3$ and $\mathrm{H} 4$ expression. Results of acetylated histone H3 and H4 expression in endometrial stromal cells (ESCs) and normal endometrial stromal cells (NESCs) are shown. Densitometric analysis of (B) histone H3 and (C) histone H4. Each column represents the mean $\pm \mathrm{SD} ; \mathrm{n}=3$. $^{* *} \mathrm{P}<0.01$, ESCs vs. NESCs.

A

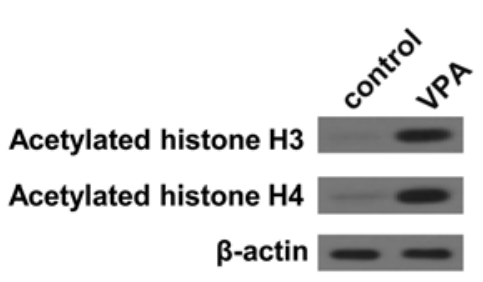

B

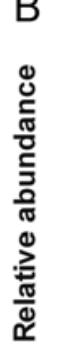

Acetylated histone $\mathrm{H} 3$

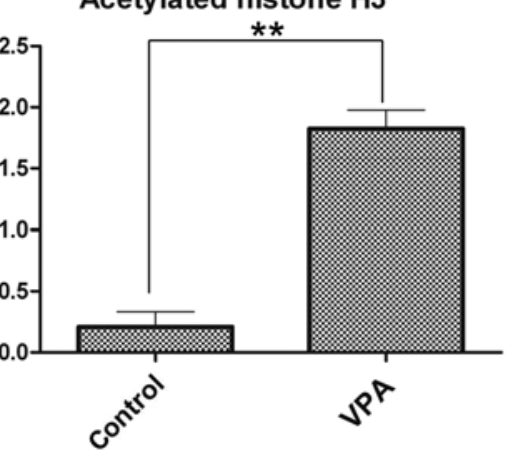

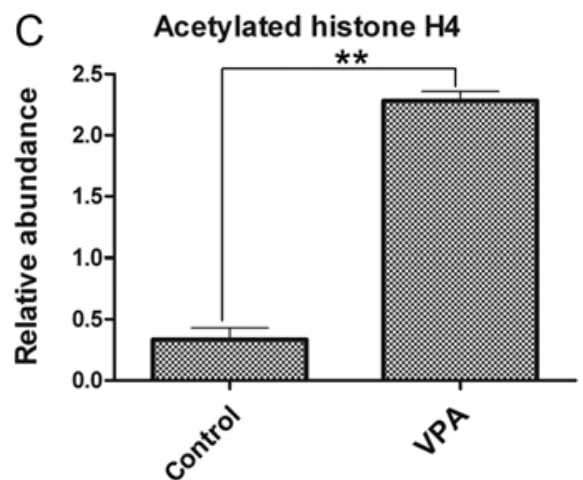

Figure 3. Effects of valproic acid (VPA) on histone acetylation. (A) Results of acetylated histone $\mathrm{H} 3$ and $\mathrm{H} 4$ expression in the control and valproic acid (VPA)-treated group. Densitometric analysis of (B) histone $\mathrm{H} 3$ and $(\mathrm{C})$ histone $\mathrm{H} 4$. Each column represents the mean $\pm \mathrm{SD} ; \mathrm{n}=3 .{ }^{* * *} \mathrm{P}<0.01$, VPA-treated group vs. control group.

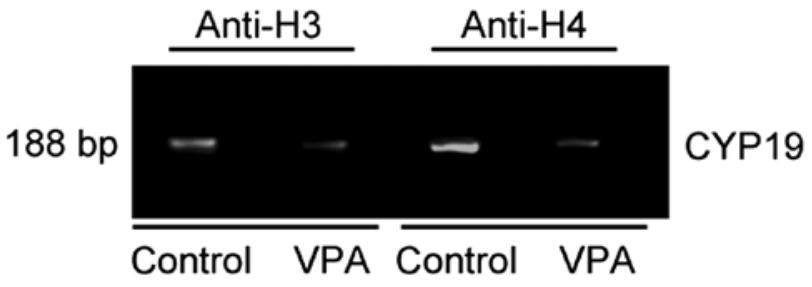

Figure 4. Effects of valproic acid (VPA) on the acetylation of the promoter region of the CYP19 gene by ChIP assay. VPA significantly decreased the amounts of PCR product, suggesting that the acetylation of histone $\mathrm{H} 3$ and $\mathrm{H} 4$ was reduced in the promoter region of the CYP19 gene inthe VPA-treated endometral stromal cells (ESCs).

sudden increase in the number of TUNEL-positive cells in both the VPA- and CYP19 siRNA-treated groups (Fig. 7B).

\section{Discussion}

This study is, to the best of our knowledge, the first to examine histone acetylation, endometriosis and CYP19 gene expression. This findings of this study may will help us to gain a better understanding of the development and treatment of endometriosis. We demonstrated in this study that VPA not only inhibited ESC viability and proliferation, and induced the apoptosis of ESCs, but also inhibited CYP19 expression, which is the encoding gene of P450 aromatase, and all of these factors promoted estrogen synthesis. Excessive estrogen production is necessary for the development of endometriosis. Hence, this study discovered a combined therapeutic target from the cellular level and histone modification at the gene level.

Local remodeling and changes in chromatin the nucleosome play a key role in the regulation of gene expression (27). One of the most important mechanisms in chromatin remodeling is protein acetylation (28). The acetylation levels of histone are controlled by the balance between HATs and HDACs. HATs transfer acetyl groups from acetyl-CoA to lysine residues in the $\mathrm{N}$-terminal region of histones (29). Conversely, HDACs remove the acetyl groups and thus restore the positive charge on lysine residues (16). As demonstrated in this study (Fig. 3), HDACis (VPA in this case) significantly promoted the acetylation of histone $\mathrm{H} 3$ and $\mathrm{H} 4$.

Prior to this study, several theories were proposed to explain the development of endometriosis, such as coelomic metaplasia (Mayer's theory), vascular and lymphatic metastasis (Halban's theory), the embryonal rest theory and the stem/progenitor cell theory $(30,31)$. The exact pathogenesis of endometriosis remains unknown; however, retrograde menstruation, proposed by Sampson (32) in 1927, is still considered the most widely accepted theory (33). According to this theory, endometrial tissues travel from the uterine cavity to the pelvic cavity through the fallopian tubes during menstrual shedding, adhere to the 
A

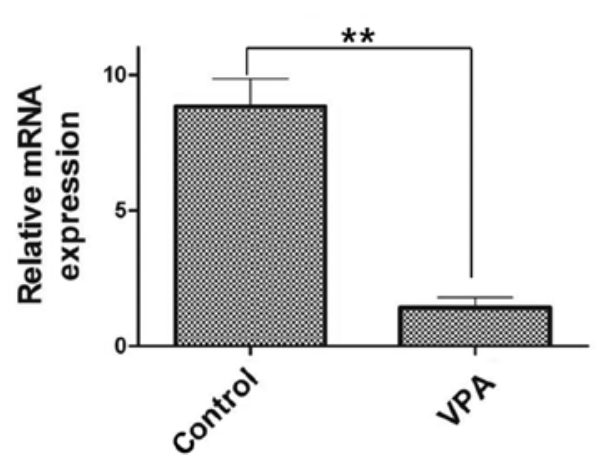

B

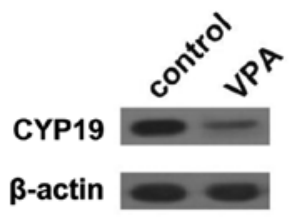

C

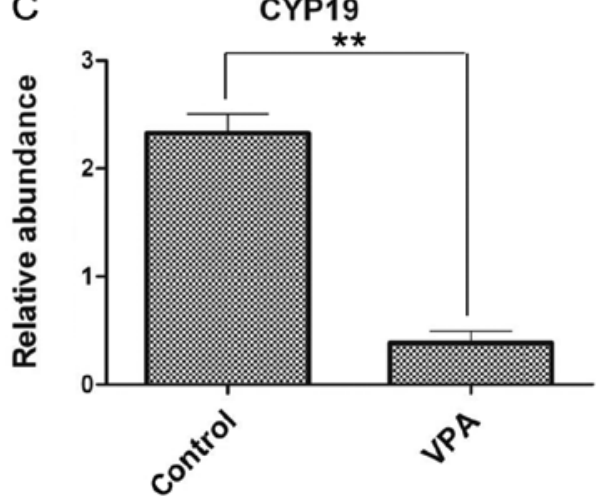

Figure 5. Effects of valproic acid (VPA) on CYP19 expression. (A) Results of RT-qPCR. Results of (B) western blot analysis and (C) densitometric analysis. Each column represents the mean $\pm \mathrm{SD} ; \mathrm{n}=3 .{ }^{* *} \mathrm{P}<0.01$, VPA-treated group vs. control group.
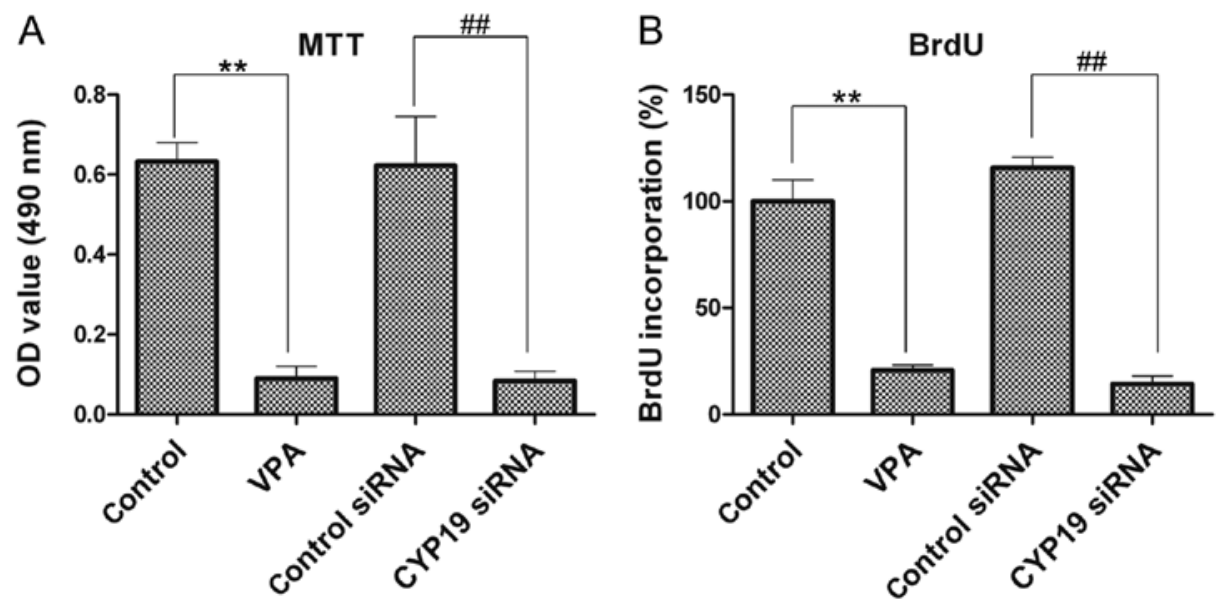

Figure 6. Effects of valproic acid (VPA) on the viability and proliferation of endometral stromal cells (ESCs). (A) MTT assay for cell viability. Data are presented as the optical density $(\mathrm{OD})$ value $(490 \mathrm{~nm})$. (B) 5-Bromo-2-deoxyuridine (BrdU) assay for cell proliferation. Data are presented as percentages relative to the values of the control groups. Each column represents the mean $\pm \mathrm{SD} ; \mathrm{n}=3 .{ }^{* *} \mathrm{P}<0.01$, VPA-treated group vs. control group; ${ }^{\# \#} \mathrm{P}<0.01$, CYP19 siRNA vs. control siRNA.
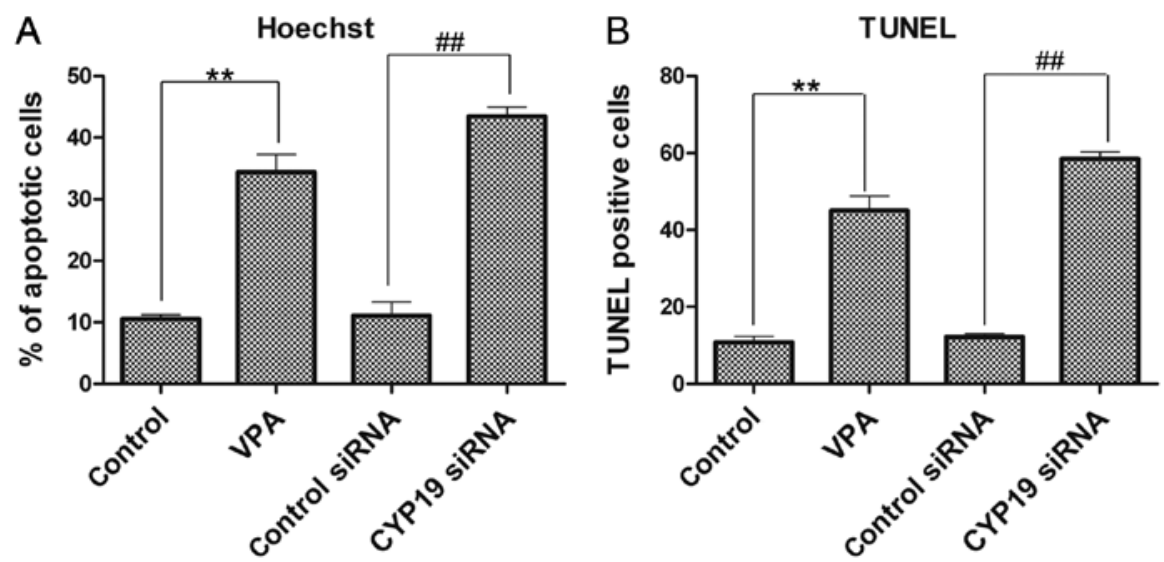

Figure 7. Effects of valproic acid (VPA) on cell apoptosis. (A) Hoechst staining. Data are presented as percentages of apoptotic cells vs. total cells. (B) TUNEL assay. Data are presented as the number of TUNEL-positive cells. Each column represents the mean $\pm \mathrm{SD} ; \mathrm{n}=3$. ** $\mathrm{P}<0.01$, VPA-treated group vs. control group; ${ }^{\# \prime} \mathrm{P}<0.01$, CYP19 siRNA vs. control siRNA.

cavity wall, invade the extracellular matrix (ECM), proliferate, and form endometrial lesions (32). It is worth noting that the incidence of retrograde menstruation is similar in women with and without endometriosis, and thus the pathogenesis seems to involve a multifactorial mechanism, which includes functionally different endometrial tissue in addition to altered immunity and other molecular abnormalities (34). Due to the high morbidity in women with dysmenorrhea or other 
gynecological diseases, and the fact that endometriosis is an estrogen-dependent disease (19), we hypothesized that the estrogen level was one of the 'multifactorial mechanisms' and thus was closely related to the development of endometriosis.

High estrogen production is a consistently observed endocrine feature of endometriosis $(19,21,35)$. The disease develops in women of reproductive age and regresses after menopause, suggesting that it is estrogen-dependent in nature. The hormone-dependent nature of the disease has prompted research on local estrogen production, with a major focus on the expression of cytochrome P450 aromatase (36). It has been demonstrated that the increased expression of CYP19 aromatase occurs in ectopically located endometrial lesions, particularly in ovarian endometriomas (37). CYP19 aromatase expression has also been detected in the eutopic endometrium of women with other uterine diseases, such as leiomyoma and adenomyosis (38). Hence, in this study, we focused on the CYP19 gene in order to investigate its association with histone acetylation.

In the present study, we found that the acetylation of histone $\mathrm{H} 3$ and $\mathrm{H} 4$ in the promoter region of the CYP19 gene was inhibited by VPA-induced histone acetylation, and thus the expression of CYP19 in the ESCs was inhibited. This seems a paradoxical result. However, it has been proven previously that histone suppresses gene expression (39). HDACis, such as VPA, play a key role in promoting the acetylation of histones closely folded with DNA, and is defined as a whole as epigenetic $(11,27)$; by contrast, VPA has a limited effect on the histones of specific genes, such as CYP19. As a result, the balance between HATs and HDACs was disrupted and the effects of HDACs remained present in histone $\mathrm{H} 3$ and $\mathrm{H} 4$ of the CYP19 gene, leading to deacetylation. Inhibition of CYP19 expression may contribute to the decreased synthesis of $\mathrm{P} 450$ aromatase, which is the rate-limiting enzyme for the pathway from androgen to estrogen, and therefore limits the synthesis of excessive estrogen. This observation may aid in the treatment of endometriosis. As previously reported, the acetylation of histones may contribute to the better treatment of endometriosis $(11,12,27)$ and aromatase inhibitors (AIs) may lead to a more effective treatment of endometriosis $(34,35)$. Hence, according to our results, VPA not only promote histone acetylation, but also acts as an AI to inhibit CYP19 gene expression, which assists with the treatment of endometriosis. VPA inhibited ESC survival and induced apoptosis, and we proved that the silencing of $C Y P 19$ had the same effects on the ESCs as VPA treatment. This indicates that VPA plays a key role in mediating $C Y P 19$ gene expression.

In conclusion, in this study, we combined the study of histone acetylation, endometriosis and the CYP19 gene. Our results provide a better and more specific therapeutic method for the treatment of endometriosis.

\section{Acknowledgements}

The present study was supported by the Shanghai Natural Science Fund (no. 12ZR1438000).

\section{References}

1. Bulun SE: Endometriosis. N Engl J Med 360: 268-279, 2009.
2. Giudice LC and Kao LC: Endometriosis. Lancet 364: 1789-1799, 2004.

3. Falcone $\mathrm{T}$ and Lebovic DI: Clinical management of endometriosis. Obstet Gynecol 118: 691-705, 2011.

4. Arya P and Shaw R: Endometriosis: Current thinking. Curr Obstet Gynaecol 15: 191-198, 2005.

5. Guo SW and Olive DL: Two unsuccessful clinical trials on endometriosis and a few lessons learned. Gynecol Obstet Invest 64: 24-35, 2007.

6. Kyama CM, Mihalyi A, Simsa P, Mwenda JM, Tomassetti C, Meuleman C and D'Hooghe TM: Non-steroidal targets in the diagnosis and treatment of endometriosis. Curr Med Chem 15: 1006-1017, 2008.

7. Goldberg AD, Allis CD and Bernstein E: Epigenetics: a landscape takes shape. Cell 128: 635-638, 2007.

8. Jaenisch R and Bird A: Epigenetic regulation of gene expression: how the genome integrates intrinsic and environmental signals. Nat Genet 33 (Suppl): 245-254, 2003.

9. Turner BM: Cellular memory and the histone code. Cell 111: 285-291, 2002.

10. Abe W, Nasu K, Nakada C, Kawano Y, Moriyama M and Narahara H: miR-196b targets c-myc and Bcl-2 expression, inhibits proliferation and induces apoptosis in endometriotic stromal cells. Hum Reprod 28: 750-761, 2013.

11. Kawano Y,Nasu K,Li H, Tsuno A, Abe W, Takai N and Narahara H: Application of the histone deacetylase inhibitors for the treatment of endometriosis: histone modifications as pathogenesis and novel therapeutic target. Hum Reprod 26: 2486-2498, 2011.

12. Nasu K, Kawano Y, Tsukamoto Y, Takano M, Takai N, Li H, Furukawa Y, Abe W, Moriyama M and Narahara H: Aberrant DNA methylation status of endometriosis: epigenetics as the pathogenesis, biomarker and therapeutic target. J Obstet Gynaecol Res 37: 683-695, 2011.

13. Huan Y, Wu Z, Zhang J, Zhu J, Liu Z and Song X: Epigenetic modification agents improve gene-specific methylation reprogramming in porcine cloned embryos. PLoS One 10: e0129803, 2015.

14. Xiaomeng X, Ming Z, Jiezhi M and Xiaoling F: Aberrant histone acetylation and methylation levels in woman with endometriosis. Arch Gynecol Obstet 287: 487-494, 2013.

15. Vialou V: Histone acetylation, gene regulation and depression. Med Sci (Paris) 26: 465-467, 2010 (In French).

16. de Ruijter AJ, van Gennip AH, Caron HN, Kemp S and van Kuilenburg AB: Histone deacetylases (HDACs): characterization of the classical HDAC family. Biochem J 370: 737-749, 2003.

17. Attar E, Tokunaga H, Imir G, Yilmaz MB, Redwine D, Putman M, Gurates B, Attar R, Yaegashi N, Hales DB and Bulun SE: Prostaglandin E2 via steroidogenic factor-1 coordinately regulates transcription of steroidogenic genes necessary for estrogen synthesis in endometriosis. J Clin Endocrinol Metab 94: 623-631, 2009.

18. Bulun SE, Fang Z, Imir G, Gurates B, Tamura M, Yilmaz B, Langoi D, Amin S, Yang S and Deb S: Aromatase and endometriosis. Semin Reprod Med 22: 45-50, 2004.

19. Bulun SE, Yang S, Fang Z, Gurates B, Tamura M and Sebastian S: Estrogen production and metabolism in endometriosis. Ann NY Acad Sci 955: 75-88, 396-406, 2002.

20. Wang L, Lu X, Wang D, Qu W, Li W, Xu X, Huang Q, Han X and Lv J: CYP19 gene variant confers susceptibility to endometriosis-associated infertility in Chinese women. Exp Mol Med 46: e103, 2014.

21. Gruber CJ, Tschugguel W, Schneeberger C and Huber JC: Production and actions of estrogens. N Engl J Med 346: 340-352, 2002.

22. Kitawaki J, Kusuki I, Koshiba H, Tsukamoto K, Fushiki S and Honjo H: Detection of aromatase cytochrome P-450 in endometrial biopsy specimens as a diagnostic test for endometriosis. Fertil Steril 72: 1100-1106, 1999.

23. Ferrero S, Remorgida V, Maganza C, Venturini PL, Salvatore S, Papaleo E, Candiani M and Leone Roberti Maggiore U: Aromatase and endometriosis: estrogens play a role. Ann NY Acad Sci 1317: 17-23, 2014.

24. Nishida M, Nasu K, Fukuda J, Kawano Y, Narahara H and Miyakawa I: Down-regulation of interleukin-1 receptor type 1 expression causes the dysregulated expression of CXC chemokines in endometriotic stromal cells: a possible mechanism for the altered immunological functions in endometriosis. J Clin Endocrinol Metab 89: 5094-5100, 2004.

25. Huang F, Zou Y, Wang $\mathrm{H}, \mathrm{Cao} \mathrm{J}$ and Yin T: In vitro apoptosis effects of GnRHII on endometrial stromal cells from patients with endometriosis. Int J Clin Exp Pathol 6: 1603-1609, 2013. 
26. Delbandi AA,Mahmoudi M,Shervin A,AkbariE,Jeddi-Tehrani M, Sankian M, Kazemnejad S and Zarnani AH: Eutopic and ectopic stromal cells from patients with endometriosis exhibit differential invasive, adhesive, and proliferative behavior. Fertil Steril 100: 761-769, 2013.

27. Takai N, Desmond JC, Kumagai T, Gui D, Said JW, Whittaker S, Miyakawa I and Koeffler HP: Histone deacetylase inhibitors have a profound antigrowth activity in endometrial cancer cells. Clin Cancer Res 10: 1141-1149, 2004.

28. Strahl BD and Allis CD: The language of covalent histone modifications. Nature 403: 41-45, 2000.

29. Kai K, Nasu K, Kawano Y, Aoyagi Y, Tsukamoto Y, Hijiya N, Abe W, Okamoto M, Moriyama M and Narahara H: Death receptor 6 is epigenetically silenced by histone deacetylation in endometriosis and promotes the pathogenesis of endometriosis. Am J Reprod Immunol 70: 485-496, 2013.

30. Amer S: Endometriosis. Obstetrics, Gynaecol Reprod Med 18: 126-133, 2008

31. Sasson IE and Taylor HS: Stem cells and the pathogenesis of endometriosis. Ann NY Acad Sci 1127: 106-115, 2008.

32. Sampson JA: Metastatic or embolic endometriosis, due to the menstrual dissemination of endometrial tissue into the venous circulation. Am J Pathol 3: 93-110 143, 1927.

33. Burney RO and Giudice LC: Pathogenesis and pathophysiology of endometriosis. Fertil Steril 98: 511-519, 2012.
34. Abu Hashim H: Potential role of aromatase inhibitors in the treatment of endometriosis. Int J Womens Health 6: 671-680, 2014.

35. Pavone ME and Bulun SE: Aromatase inhibitors for the treatment of endometriosis. Fertil Steril 98: 1370-1379, 2012.

36. Rhee HS, Oh SH, Ko BJ, Han DM, Jeon BH, Park H, Moon HB and Kim WS: Expression of 3beta-hydroxysteroid dehydrogenase and $\mathrm{P} 450$ side chain cleavage enzyme in the human uterine endometrium. Exp Mol Med 35: 160-166, 2003.

37. Bukulmez O, Hardy DB, Carr BR, Auchus RJ, Toloubeydokhti T, Word RA and Mendelson CR: Androstenedione up-regulation of endometrial aromatase expression via local conversion to estrogen: potential relevance to the pathogenesis of endometriosis. J Clin Endocrinol Metab 93: 3471-3477, 2008.

38. Wölfler MM, Nagele F, Kolbus A, Seidl S, Schneider B, Huber JC and Tschugguel W: A predictive model for endometriosis. Hum Reprod 20: 1702-1708, 2005.

39. Verdone L, Caserta M and Di Mauro E: Role of histone acetylation in the control of gene expression. Biochem Cell Biol 83: 344-353, 2005 